\title{
Immunohistochemical pitfalls and the importance of glypican 3 and arginase in the diagnosis of scirrhous hepatocellular carcinoma
}

\author{
Gregor Krings ${ }^{1}$, Rageshree Ramachandran ${ }^{1}$, Dhanpat Jain ${ }^{2,7}$, Tsung-Teh $\mathrm{Wu}^{3,7}$, \\ Matthew M Yeh ${ }^{4,7}$, Michael Torbenson ${ }^{5,7}$ and Sanjay Kakar,6,7 \\ ${ }^{1}$ Department of Pathology, University of California, San Francisco, San Francisco, CA, USA; ${ }^{2}$ Department of \\ Pathology, Yale University, New Haven, CT, USA; ${ }^{3}$ Department of Laboratory Medicine and Pathology, \\ Mayo Clinic, Rochester, MN, USA; ${ }^{4}$ Department of Pathology, University of Washington, Seattle, WA, USA; \\ ${ }^{5}$ Department of Pathology, Johns Hopkins University, Baltimore, MD, USA and ${ }^{6}$ Department of Pathology, \\ Veterans Affairs Medical Center, San Francisco, CA, USA
}

\begin{abstract}
Scirrhous hepatocellular carcinoma is a rare ill-defined morphological subtype of hepatocellular carcinoma characterized by marked stromal fibrosis. This variant can be difficult to distinguish from intrahepatic cholangiocarcinoma and metastatic adenocarcinoma, especially on needle biopsies. We performed immunohistochemistry for hepatocellular and adenocarcinoma-associated markers on 20 scirrhous hepatocellular carcinoma cases and compared the results with classical hepatocellular carcinoma and intrahepatic cholangiocarcinoma. Scirrhous hepatocellular carcinomas were significantly less likely to be HepPar-1 positive than classical hepatocellular carcinomas $(26 \%$ and $74 \%$, respectively; $\boldsymbol{P}<0.001)$ and were significantly more likely to express adenocarcinoma-associated markers such as epithelial cell adhesion molecule (63 vs 11\%; $P<0.001$ ), cytokeratin 19 (26 vs $2 \% ; P<0.001$ ), and cytokeratin 7 (53 vs $2 \% ; P<0.001)$. At least one of these adenocarcinoma-related markers was positive in $\mathbf{8 0} \%$ of scirrhous hepatocellular carcinoma cases. Glypican 3 and arginase were positive in $79 \%$ and $\mathbf{8 5 \%}$ of cases of scirrhous hepatocellular carcinoma, respectively; the combined use of these two markers yielded $100 \%$ sensitivity for scirrhous hepatocellular carcinoma. In conclusion, the scirrhous morphology, absence of HepPar-1 staining, and frequent positivity with adenocarcinoma-related markers in scirrhous hepatocellular carcinoma can lead to an erroneous diagnosis of adenocarcinoma. Glypican 3 and arginase are the most reliable markers for identifying hepatocellular differentiation in this setting.
\end{abstract}

Modern Pathology (2013) 26, 782-791; doi:10.1038/modpathol.2012.243; published online 25 January 2013

Keywords: arginase; cholangiocarcinoma; glypican 3; scirrhous hepatocellular carcinoma

Scirrhous hepatocellular carcinoma is a rare morphological subtype of hepatocellular carcinoma, comprising $<5 \%$ of all hepatocellular carcinomas. ${ }^{1-5}$ In addition to marked stromal fibrosis, scirrhous hepatocellular carcinomas are often characterized by subcapsular location, contiguous multinodularity, lack of encapsulation, lack of necrosis or

Correspondence: Dr Sanjay Kakar, MD, Department of Anatomic Pathology, San Francisco Veterans Affairs Medical Center, 4150 Clement Street, San Francisco, CA 94121, USA.

E-mail: sanjay.kakar@ucsf.edu

${ }^{7}$ GREMLINS Liver Study Group.

Received 1 October 2012; revised 15 December 2012; accepted 17 December 2012; published online 25 January 2013 hemorrhage, clear-cell change or hyaline bodies, and preserved intratumoral portal tracts. ${ }^{2,3}$ Radiological studies in scirrhous hepatocellular carcinomas often show atypical findings such as peripheral enhancement in the arterial phase and persistent enhancement in the venous phase. ${ }^{4,6}$

Immunophenotypically, hepatocellular markers such as HepPar-1 are negative in the majority of scirrhous hepatocellular carcinomas, whereas markers such as cytokeratin 7 (CK7), which is typically associated with adenocarcinoma, are often positive, ${ }^{2}$ thereby further confounding the diagnostic dilemma. The combination of atypical radiological features, abundant fibrous stroma, and aberrant immunohistochemical features can easily lead to a 
mistaken diagnosis of intrahepatic cholangiocarcinoma, metastatic adenocarcinoma, or combined hepatocellular-cholangiocarcinoma.

Distinction of scirrhous hepatocellular carcinoma from intrahepatic cholangiocarcinoma and metastatic adenocarcinoma has important therapeutic and prognostic implications. Long-term follow-up studies suggest that scirrhous hepatocellular carcinoma may be associated with similar or better prognosis than classical hepatocellular carcinoma. ${ }^{2,4,5,7}$ In contrast, prognosis and survival of patients with intrahepatic cholangiocarcinoma is significantly worse than hepatocellular carcinoma. ${ }^{5,8,9}$ Lymph node dissection is routinely performed during resection of intrahepatic cholangiocarcinoma but not hepatocellular carcinoma, as lymph node metastasis is relatively uncommon in the latter. ${ }^{10,11}$ Treatment options for unresectable hepatocellular carcinoma include sorafenib and intra-arterial chemoembolization, whereas chemotherapeutic agents such as gemcitabine and fluoropyramidines are not beneficial.12,13 In contrast, unresectable intrahepatic cholangiocarcinomas or resectable tumors with positive margins are treated with gemcitabine- or fluoropyramidinebased regimens. ${ }^{14}$ Finally, orthotopic liver transplantation is established as a potentially curative option for selected cirrhotic patients with hepatocellular carcinoma who meet the Milan or extended UCSF criteria, whereas intrahepatic cholangiocarcinoma is considered a contraindication for orthotopic liver transplantation in most centers becasue of high recurrence rate and poor overall outcome. ${ }^{15-18}$

Despite the clinical relevance of accurate diagnosis, the utility of hepatocellular markers other than HepPar-1, such as polyclonal antibody against carcinoembryonic antigen (CEA) or expression of glypican 3 (GPC3) and arginase (ARG1), has not been studied in scirrhous hepatocellular carcinoma. Both HepPar-1 and polyclonal antibody against CEA show low sensitivity for poorly differentiated hepatocellular carcinoma. ${ }^{19-22}$ In contrast, GPC3, an oncofetal antigen that is expressed in $>80 \%$ of hepatocellular carcinomas, has high sensitivity for poorly differentiated hepatocellular carcinoma. ${ }^{22-24}$ ARG1, a manganese metalloenzyme active in the urea cycle, has recently been identified as a sensitive and specific hepatocellular marker, ${ }^{25}$ and also has not been evaluated in scirrhous hepatocellular carcinoma. Similarly, whereas CK7 positivity has been reported in scirrhous hepatocellular carcinoma, ${ }^{2}$ the expression of other adenocarcinoma-associated markers, such as epithelial cell adhesion molecule (EPCAM) and cytokeratin 19 (CK19), has not been explored in scirrhous hepatocellular carcinoma. This study examines the immunophenotypic characteristics of scirrhous hepatocellular carcinoma using a variety of hepatocellularand adenocarcinoma-associated markers, and compares the results with classical hepatocellular carcinoma and intrahepatic cholangiocarcinoma.

\section{Materials and methods}

The study population comprised 20 cases of scirrhous hepatocellular carcinoma and 16 cases of intrahepatic cholangiocarcinoma from the authors' institutions. Scirrhous hepatocellular carcinoma was defined as hepatocellular carcinoma with fibrous stroma comprising at least $50 \%$ of one lowpower field. Scirrhous hepatocellular carcinoma cases included 11 resection specimens, 1 explant, and 8 core needle biopsies. In all biopsies, seven resection specimens and one explant specimen, the tumor showed abundant fibrous stroma that comprised $>50 \%$ of the tumor. In four resection specimens, the scirrhous component was admixed with classical hepatocellular carcinoma. None of the cases had received preoperative chemotherapy or radiation. Cases of fibrolamellar carcinoma were excluded.

All specimens were fixed in $10 \%$ buffered formalin and embedded in paraffin. For immunohistochemistry, the following antibodies were used: hepatocyte-specific antigen (HepPar-1; dilution 1:200, clone OCH1E5; DAKO, Glostrup, Denmark), GPC3 (undiluted, clone 1G12; Biomosaics, Burlington, VT, USA), ARG1 (1:400 dilution, polyclonal; Sigma-Aldrich, St Louis, MO, USA), polyclonal antibody against CEA (1:20 dilution, polyclonal; DAKO, Glostrup, Denmark), CD117/c-kit (1:200 dilution; DAKO), CK7 (1:500 dilution, clone OV-TL 12/30; DAKO), CK19 (1:60 dilution, clone RCK108; DAKO), EPCAM (1:160 dilution, clone MOC-31; DAKO), and CD56 (1:100 dilution, clone 123C3; Zymed, San Francisco, CA, USA). DAKO 6.0 automated antigen retrieval system was used for all immunohistochemistry except ARG1 (ER1 antigen retrieval), CD56 (TRS 9.0 antigen retrieval), and CK19 (no antigen retrieval).

The intensity of immunohistochemical staining was scored as 0 (negative), $1+$ (weak), $2+$ (moderate), and $3+$ (strong). The number of positive cells was recorded as focal $(<10 \%)$, patchy $(10-50 \%)$, or diffuse $(>50 \%)$. A score of at least $2+$ staining in $\geq 10 \%$ of cells was regarded as positive. The results were compared with 169 cases of classical hepatocellular carcinoma (64 well differentiated, 91 moderately differentiated, and 14 poorly differentiated). ${ }^{26}$ In some classical hepatocellular carcinoma cases, the available tissue was insufficient to perform all of the stains, and this is noted where applicable. The statistical analysis of differences in immunohistochemical staining patterns between groups was performed using Fisher's exact test and $\chi^{2}$ test.

\section{Results}

\section{Study Population}

The age at diagnosis of scirrhous hepatocellular carcinoma patients ranged from 26 to 70 years (mean 56.5 years). There were 12 men and 8 women. 
Cirrhosis was present in nine $(45 \%)$ cases. Two patients each had chronic hepatitis B or C, and steatosis or steatohepatitis was seen in seven cases. Serum $\alpha$-fetoprotein level was available in 12 cases at diagnosis and was elevated in six patients. Scirrhous hepatocellular carcinoma cases were enriched for background fatty liver disease compared with classical hepatocellular carcinoma (Table 1).

\section{Hepatocellular Markers in Scirrhous and Classical HCC}

In addition to fibrous stroma, all scirrhous hepatocellular carcinoma cases displayed at least focal 'hepatoid' morphology (polygonal tumor cells with moderate amounts of cytoplasm and lack of gland formation or mucin production; Figure 1). HepPar-1 was positive in $26 \%$ of scirrhous hepatocellular carcinoma compared with $74 \%$ of classical hepatocellular carcinoma $(P<0.001$; Table 2 and Figure 2). In the four cases of mixed scirrhous and classical hepatocellular carcinoma, both components were positive for HepPar-1 in one case, and both areas were negative for HepPar-1 in one case. In the remaining two cases with admixed features, HepPar-1 staining was seen in classical areas only (Figure 3). There was no significant difference in canalicular staining of polyclonal antibody against CEA in scirrhous and classical hepatocellular carcinoma (37\% vs $54 \%$, respectively; $P=0.223$ ).

GPC3 staining results were available in 19 scirrhous hepatocellular carcinoma cases. Of these, $15(79 \%)$ were positive for GPC3, with strong diffuse staining in $9(47 \%)$ tumors (Table 2 and Figure 2). The results were similar to classical hepatocellular carcinoma, of which $69 \%$ were positive for GPC3 $(P=0.440)$. In the four cases of admixed scirrhous and classical hepatocellular carcinoma, both components were positive for GPC3 in two cases. In the other two cases, GPC3 was positive only in the scirrhous component (Figure 3).

ARG1 staining results were available in 13 scirrhous hepatocellular carcinoma cases. Of these, ARG1 was expressed in 11 (85\%) cases, with strong diffuse staining in $9(69 \%)$ cases (Table 2 and Figure 2). The results were comparable to classical hepatocellular carcinoma, which showed ARG1 expression in $95 \%$ of cases $(P=0.189)$. In all four tumors with admixed scirrhous and classical hepatocellular carcinoma, ARG1 staining was positive in both components (Figure 3). All scirrhous hepatocellular carcinoma cases showed positive staining for either GPC3 and/or ARG1.

\section{CK7, CK19, and EPCAM in Scirrhous and Classical HCC}

CK7 and CK19 were positive in $53 \%$ and $26 \%$ of scirrhous hepatocellular carcinoma cases compared with $2 \%$ and $2 \%$ of classical hepatocellular carcinoma, respectively $(P<0.001$ for both; Table 2 and Figure 2). Staining for EPCAM yielded similar results $(63 \%$ scirrhous hepatocellular carcinoma vs $11 \%$ classical hepatocellular carcinoma; $P<0.001$ ). Positive staining for at least one of these markers was seen in $80 \%$ of scirrhous hepatocellular carcinoma, whereas two or more markers were positive in $45 \%$ of cases. Among the 14 scirrhous hepatocellular carcinoma cases that were HepPar-1 negative, 64\% expressed at least two adenocarcinoma-associated markers, and similar results were seen in $60 \%$ of CEA-negative scirrhous hepatocellular carcinoma.

Of the four cases showing admixed scirrhous and classical hepatocellular carcinoma, one case showed expression of CK7 only and another showed expression of both CK7 and CK19 in the scirrhous but not the classical component, whereas the other two cases expressed at least one of the adenocarcinomaassociated markers in both regions (Figure 3).

\section{Immunophenotypic Profile of Intrahepatic Cholangiocarcinoma}

All $16(100 \%)$ cases of intrahepatic cholangiocarcinoma expressed CK7 and EPCAM, and 15 (94\%) also expressed CK19 (Table 2). The results were significantly different from classical hepatocellular carcinoma $(2 \%, 11 \%$, and $2 \%$, respectively; $P<0.001$ for each) as well as scirrhous hepatocellular carcinoma $(53 \%, 63 \%$, and $26 \%$, respectively; Table 2). The numbers for scirrhous hepatocellular carcinoma are intermediate between intrahepatic cholangiocarcinoma and classical hepatocellular carcinoma. HepPar-1 and GPC3 positivity was seen

Table 1 Clinical features of scirrhous and classical hepatocellular carcinoma

\begin{tabular}{|c|c|c|c|c|c|c|}
\hline & $\begin{array}{c}\text { Gender } \\
(M: F)\end{array}$ & $\begin{array}{l}\text { Mean age in } \\
\text { years (range) }\end{array}$ & $\begin{array}{c}\text { Hepatitis } \\
B(\%)\end{array}$ & $\begin{array}{c}\text { Hepatitis } \\
C(\%)\end{array}$ & $\begin{array}{c}\text { Steatosis or } \\
\text { steatohepatitis } \\
(\%)\end{array}$ & $\begin{array}{c}\text { Cirrhosis } \\
(\%)\end{array}$ \\
\hline Scirrhous hepatocellular carcinoma ${ }^{\mathrm{a}}(n=20)$ & $1.5: 1$ & $56.5(26-70)$ & 12 & 12 & 41 & 45 \\
\hline Classical hepatocellular carcinoma ${ }^{\mathrm{b}}(n=169)$ & $2.5: 1$ & $59.2(11-85)$ & 24 & 59 & 7 & $74^{\mathrm{C}}$ \\
\hline$P$-value $\quad P$ - & 0.306 & 0.400 & 0.362 & $<0.001$ & $<0.001$ & 0.016 \\
\hline
\end{tabular}

${ }^{a}$ Information for cirrhosis risk factors available in 17 cases.

${ }^{b}$ Information for cirrhosis risk factors available in 129 cases.

${ }^{\mathrm{C}}$ Information available in 163 cases. 

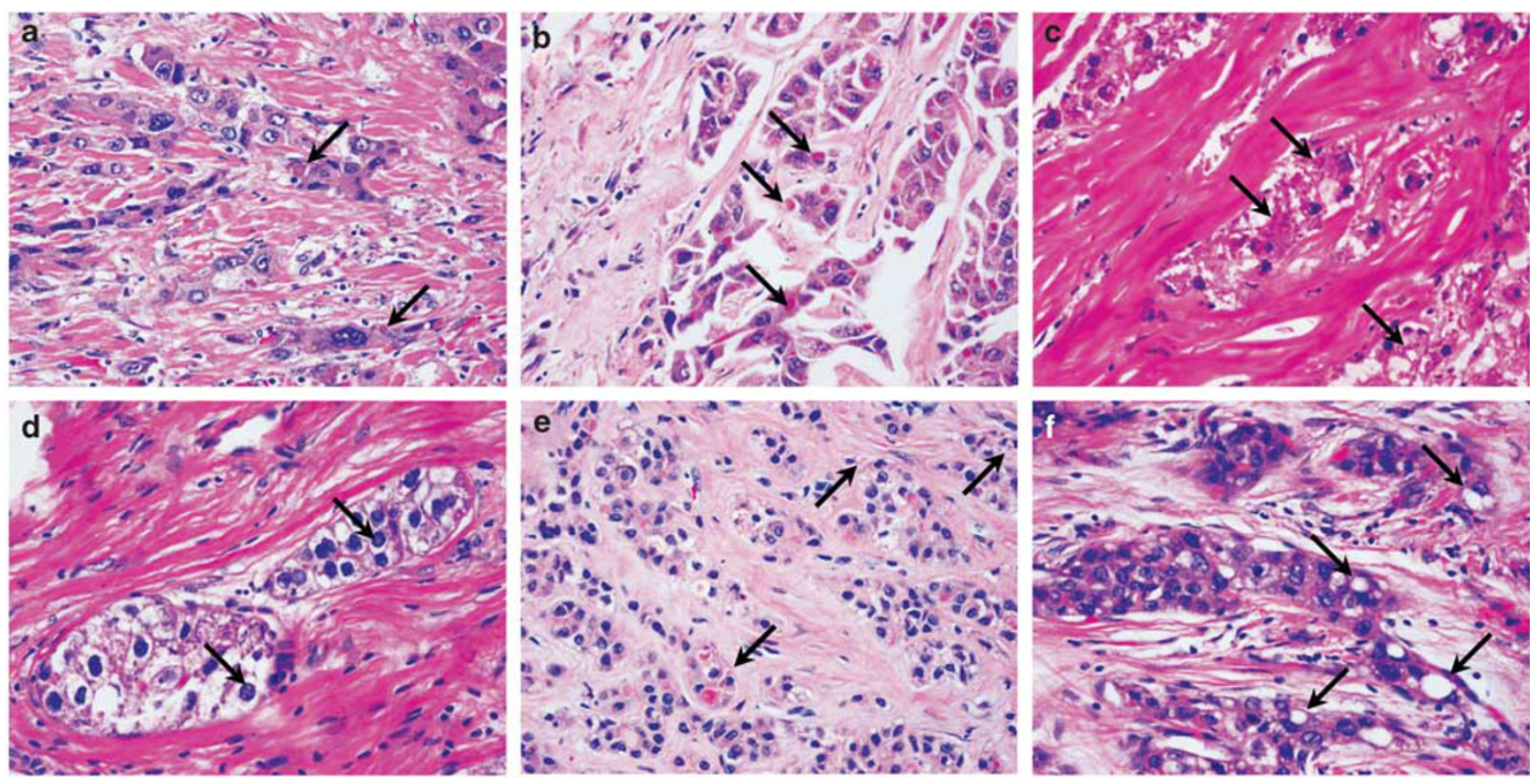

Figure 1 Morphological features of scirrhous hepatocellular carcinoma. Representative examples of scirrhous hepatocellular carcinoma illustrating the 'hepatoid' morphology (polygonal tumor cells with moderate cytoplasm), abundant fibrous stroma, and lack of glandular differentiation or mucin production (hematoxylin-eosin stain, $\times 400$ ). Additional findings (eg, arrows) include intracytoplasmic eosinophilic globules (a, b), Mallory hyaline (c), intranuclear pseudoinclusions (d), bile (e), and cytoplasmic fat (f).

Table 2 Immunohistochemical staining of scirrhous hepatocellular carcinoma, classical hepatocellular carcinoma, and intrahepatic cholangiocarcinoma

\begin{tabular}{|c|c|c|c|c|c|c|c|}
\hline & $C E A^{\mathrm{a}}$ & HepPar-1 & GPC3 & $A R G 1$ & CK7 & CK19 & EPCAM \\
\hline Scirrhous hepatocellular carcinoma $(n=20)^{\mathrm{b}}$ & 37 & 26 & 79 & 85 & 53 & 26 & 63 \\
\hline Classical hepatocellular carcinoma $(n=169)^{\mathrm{b}}$ & 54 & 74 & 69 & 95 & 2 & 2 & 11 \\
\hline Cholangiocarcinoma $(n=16)^{\mathrm{b}}$ & 0 & 7 & 6 & 0 & 100 & 94 & 100 \\
\hline$P$-value (scirrhous vs classical hepatocellular carcinoma) & 0.223 & $<0.001$ & 0.440 & 0.189 & $<0.001$ & $<0.001$ & $<0.001$ \\
\hline$P$-value (scirrhous hepatocellular carcinoma vs cholangiocarcinoma) & 0.026 & 0.209 & $<0.001$ & $<0.001$ & 0.001 & 0.001 & 0.011 \\
\hline
\end{tabular}

Abbreviations: ARG1, arginase; CEA, polyclonal antibody to carcinoembryonic antigen; CK7, cytokeratin 7; CK19, cytokeratin 19; EPCAM, epithelial cell adhesion molecule; GPC3, glypican 3.

Numbers reflect percentages.

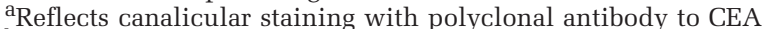

bIn some cases, available tissue was insufficient to perform all stains. For classical hepatocellular carcinoma: CEA, $n=150$; GPC3, $n=153$; HepPar-1 and ARG1, $n=147$; CK7, $n=158$; CK19, $n=152$; EPCAM, $n=149$. For scirrhous hepatocellular carcinoma: CEA, HepPar-1, GPC3, CK7, CK19, and EPCAM, $n=19$; ARG1, $n=13$. For cholangiocarcinoma: CEA, $n=12$, HepPar-1, $n=14$.

in one case of intrahepatic cholangiocarcinoma each, whereas canalicular CEA and ARG1 were negative in all cases.

\section{Progenitor Cell-Associated Markers in Scirrhous HCC}

Immunohistochemistry for CD117/c-kit and CD56 was performed on a limited number of scirrhous hepatocellular carcinoma cases $(n=5$ and 4 , respectively). One case showed focal weak CD117 staining, wheres CK7, CK19, EPCAM, and CD56 were negative in these areas. Patchy CD56 staining was seen in one other case, whereas CK7, CK19, EPCAM, and CD117 were negative in this case. Neither of these two cases showed small tumor cells at the periphery of the cell nests, and the CD117 and CD56 staining was observed in randomly distributed tumor cells (Figure 4a-C).

\section{Discussion}

The distinction of scirrhous hepatocellular carcinoma from intrahepatic cholangiocarcinoma and combined hepatocellular-cholangiocarcinoma carries important prognostic implications and can influence the surgical approach, choice of chemotherapy, and decision to perform liver transplantation. ${ }^{10-18}$ The World Health Organization (WHO) defines scirrhous hepatocellular carcinoma as a variant of hepatocellular carcinoma with marked sinusoidal fibrosis and atrophy of tumor trabeculae. ${ }^{5}$ This description does not provide a precise definition of 
scirrhous hepatocellular carcinoma. Most studies have used a fibrous stromal component of $>50 \%$ to define scirrhous hepatocellular carcinoma, but other definitions, such as $>25 \%$ fibrous stroma and/or a combination of gross and microscopic features, have been used. ${ }^{1-4}$ Owing to the clinical implications involved, the correct categorization of this tumor as a hepatocellular carcinoma variant is more important than determining an exact cutoff for the amount of fibrous stroma requisite for the diagnosis.

Our study shows that the commonly utilized hepatocellular marker HepPar-1 has a significantly lower rate of positivity in scirrhous hepatocellular carcinoma compared with classical hepatocellular carcinoma. The low sensitivity of HepPar-1 in scirrhous hepatocellular carcinoma has been previously described in isolated reports, ${ }^{27,28}$ as well as in two case series, in which sensitivities of 43 and $80 \%$ were reported, and were lower than classical hepatocellular carcinoma. ${ }^{2,7}$ A canalicular pattern of staining with polyclonal antibody against CEA is considered a specific marker of hepatocellular differentiation but has low sensitivity. ${ }^{19-21}$ Isolated case reports show variable expression in a limited number of scirrhous hepatocellular carcinoma cases. $^{27}$ In our study, canalicular CEA expression was seen in $37 \%$ and $54 \%$ of scirrhous and classical hepatocellular carcinomas, respectively. Its utility as a diagnostic marker in this context is therefore limited by the low sensitivity. In this respect, scirrhous hepatocellular carcinoma is similar to poorly differentiated hepatocellular carcinoma, where both HepPar-1 and CEA have relatively lower sensitivity. Our study also shows that the expression of adenocarcinoma-associated markers, such as CK7, CK19, and EPCAM, is significantly higher in scirrhous hepatocellular carcinoma compared with classical hepatocellular carcinoma. Matsuura et $a l^{2}$ also reported positive CK7 staining in $71 \%$ of scirrhous hepatocellular carcinoma compared with $16 \%$ of classical hepatocellular carcinoma, although CK19 was negative in all
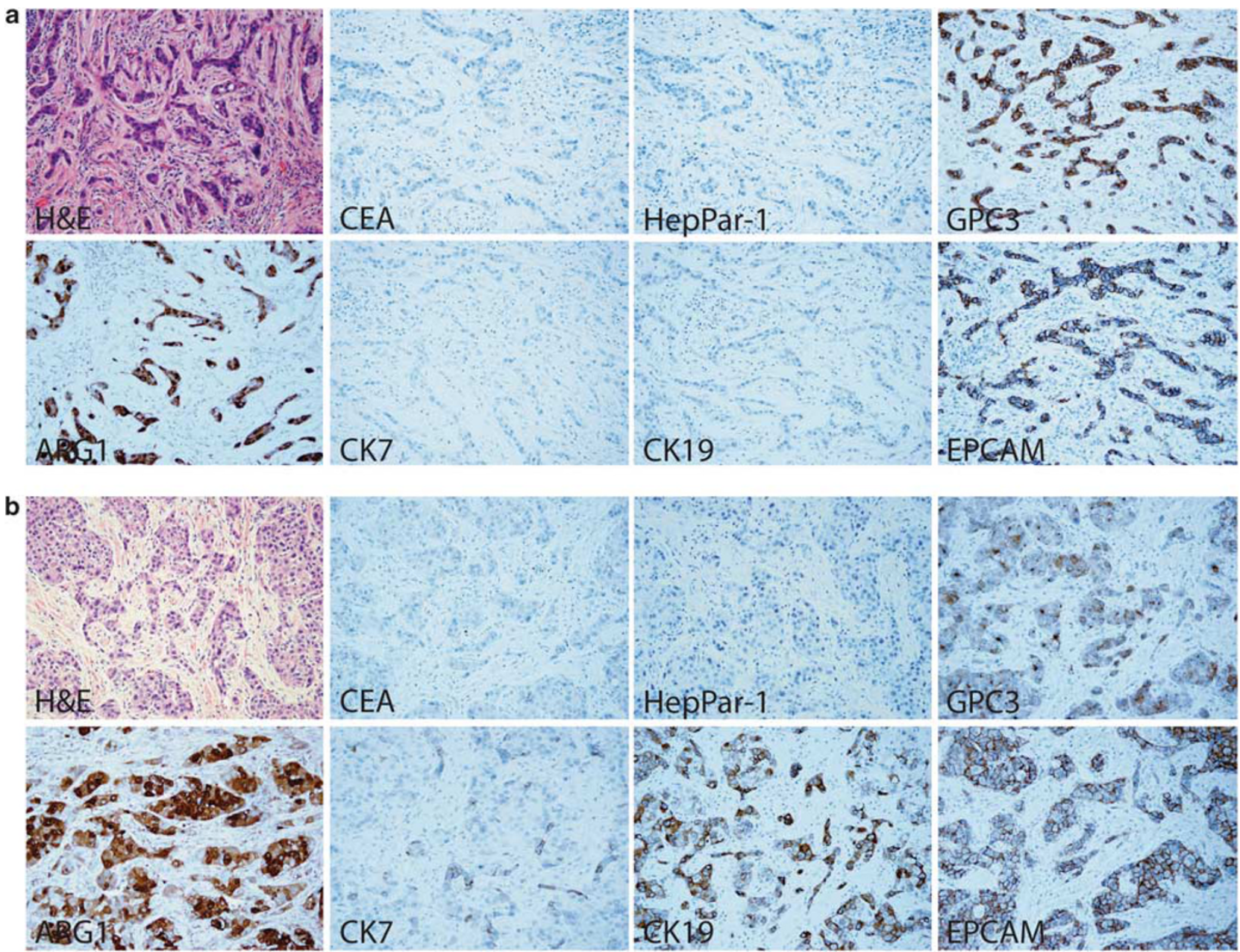

Figure 2 Immunohistochemical staining patterns of scirrhous hepatocellular carcinoma. (a) Positive staining for glypican 3 (GPC3), arginase (ARG1), and epithelial cell adhesion molecule (EPCAM); HepPar-1, carcinoembryonic antigen (CEA), cytokeratin 7 (CK7) and CK19 are negative $(\times 200)$. (b) Positive staining for glypican 3, arginase, EPCAM, CK19, and CK7 (patchy); HepPar-1 and CEA are negative $(\times 200)$. (c) Positive staining for arginase, CK7, glypican 3 (patchy), and EPCAM (patchy); HepPar-1, CEA, and CK19 are negative $(\times 200)$. (d) Positive staining for glypican 3, arginase, and EPCAM; HepPar-1, CEA, CK7, and CK19 are negative $(\times 200)$. 

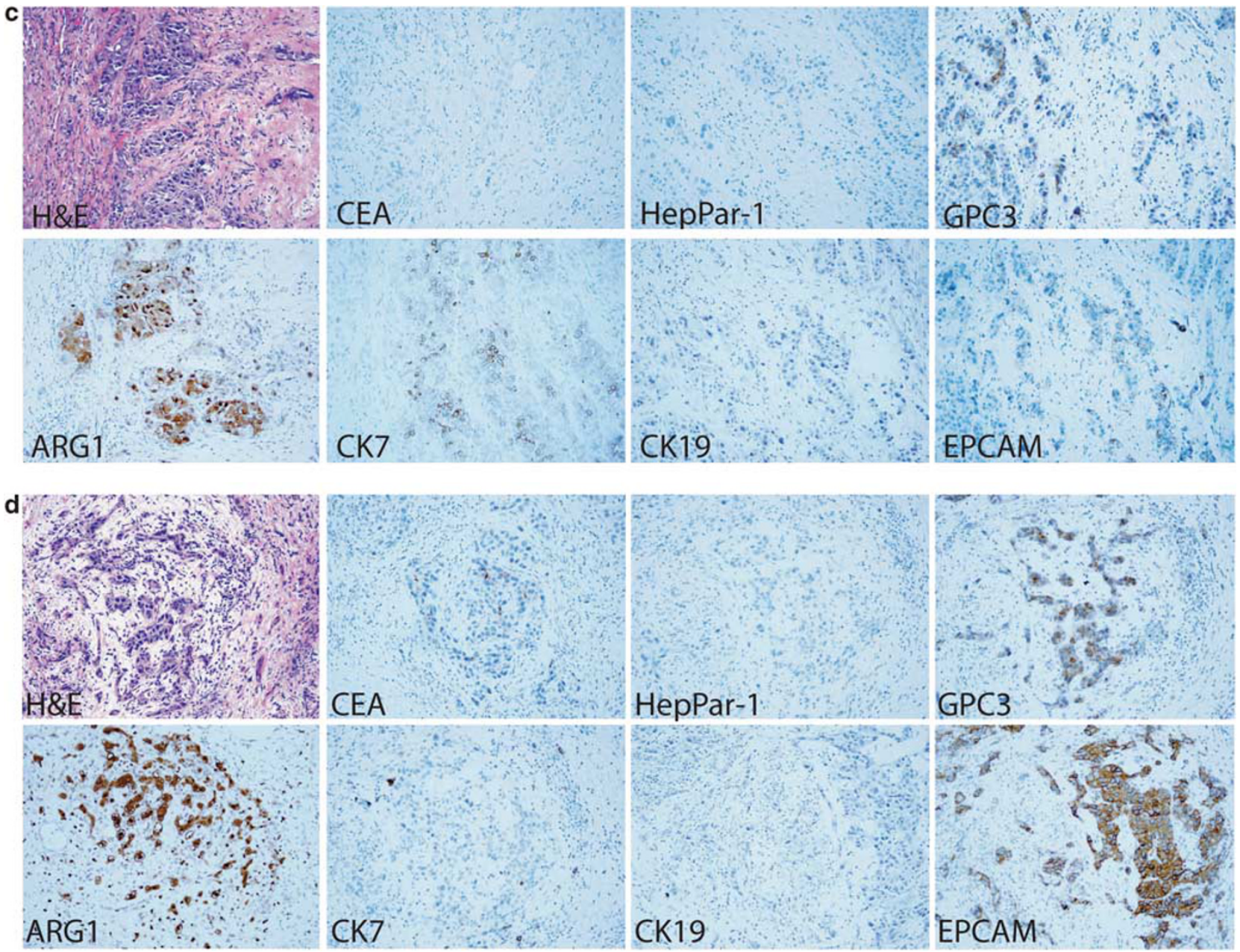

Figure 2 Continued.

scirrhous hepatocellular carcinoma cases in that study. Kurogi et $a l^{3}$ also reported negative CK19 (but positive HepPar-1) staining in all scirrhous hepatocellular carcinomas in their study population, whereas Ariizumi et $a l^{27}$ reported CK19 positivity in two scirrhous hepatocellular carcinoma cases. These differences may reflect differences in study populations and/or biological heterogeneity. CK7 and CK19 expression is less common in classical hepatocellular carcinoma and was expressed in $12 \%$ and $6 \%$ of cases, respectively, in a large study. ${ }^{29}$

While HepPar-1 and polyclonal antibody against CEA offer limited utility for diagnosis of scirrhous hepatocellular carcinoma, the role of more recently described hepatocellular markers, such as GPC3 and ARG1, has not been systematically examined. GPC3 is an oncofetal protein, which is expressed in $>80 \%$ of hepatocellular carcinomas and has higher sensitivity than HepPar-1 and CEA for poorly differentiated hepatocellular carcinoma. ${ }^{22-24,30-38}$ GPC3 is not a specific marker for hepatocellular differentiation and is expressed in a variety of other tumors, most of which do not share clinical or morphological resemblance to hepatocellular carcinoma. ${ }^{39}$ Expression in cholangiocarcinoma has been described but is rare $(<5 \%$ based on combined results of five studies including the present). ${ }^{39-42}$ GPC3 expression was seen in $79 \%$ of scirrhous hepatocellular carcinoma and 6\% (1 case) of intrahepatic cholangiocarcinoma in our study. Hence, GPC3 expression favors hepatocellular carcinoma over intrahepatic cholangiocarcinoma, even though it is not entirely specific in this context. ARG1 expression has higher sensitivity than HepPar-1 across the differentiation spectrum of hepatocellular carcinoma. ${ }^{25}$ Arginase staining may rarely be seen in other tumors but has not been described in cholangiocarcinoma. ${ }^{25,43}$ Positive arginase staining was observed in $85 \%$ of scirrhous hepatocellular carcinomas and none of the cholangiocarcinomas in our study. Notably, the combined use of GPC3 and arginase identified all cases of scirrhous hepatocellular carcinoma.

The gene expression pattern in scirrhous hepatocellular carcinoma has similarities to intrahepatic cholangiocarcinoma. ${ }^{44}$ Along with the expression of adenocarcinoma-associated markers and concomitant absence of hepatocellular markers such as HepPar-1 and CEA, this may raise the argument that 

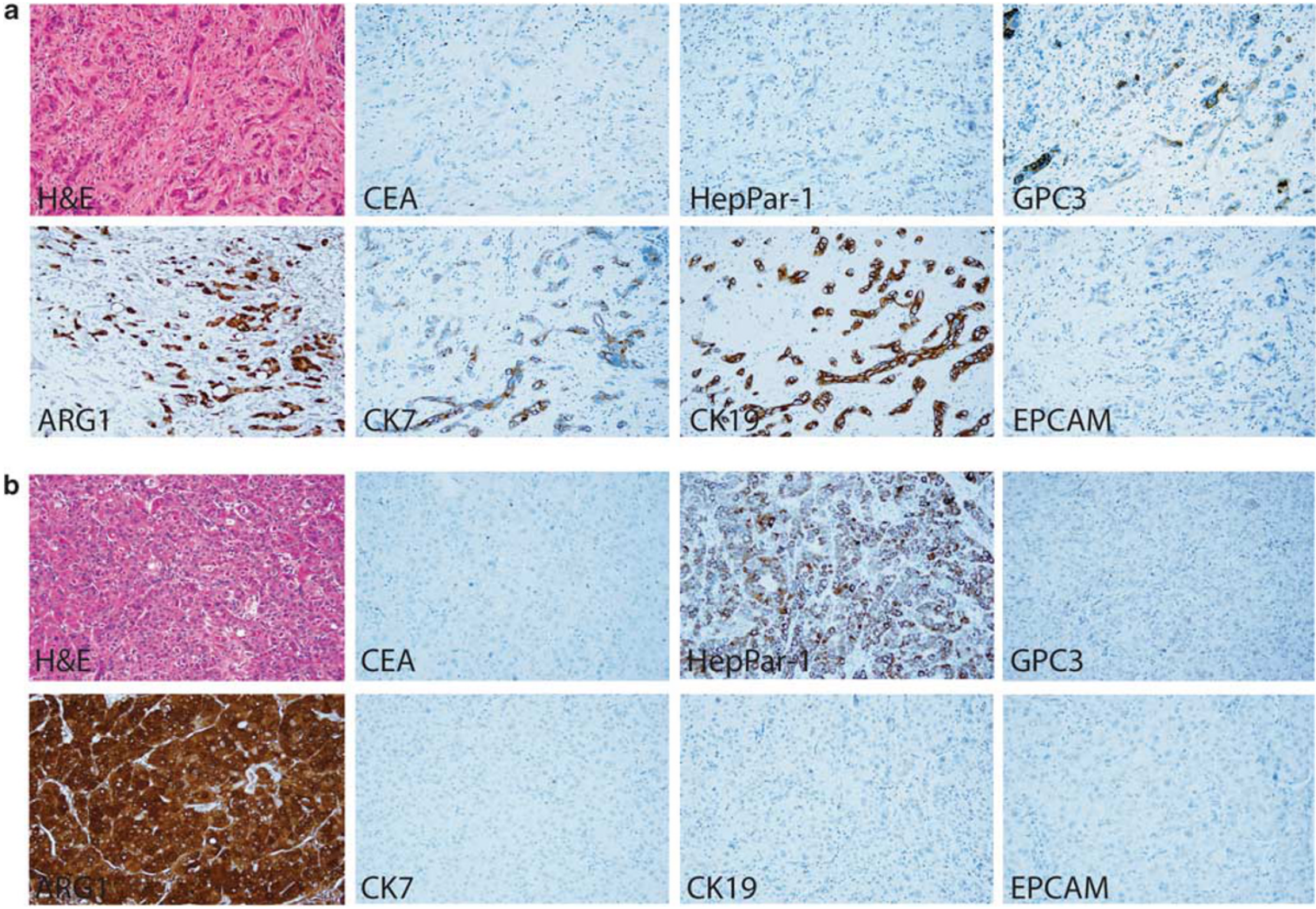

Figure 3 Immunohistochemical staining patterns of admixed scirrhous-classical hepatocellular carcinoma. The scirrhous hepatocellular carcinoma portion (a) is negative for HepPar-1 and positive for glypican 3 (GPC3), cytokeratin 7 (CK7), and CK19, whereas classical hepatocellular carcinoma (b) is positive for HepPar-1 and negative for glypican 3, CK7, and CK19. Arginase (ARG1) is expressed in both areas, while carcinoembryonic antigen (CEA) and epithelial cell adhesion molecule (EPCAM) are negative in both components.
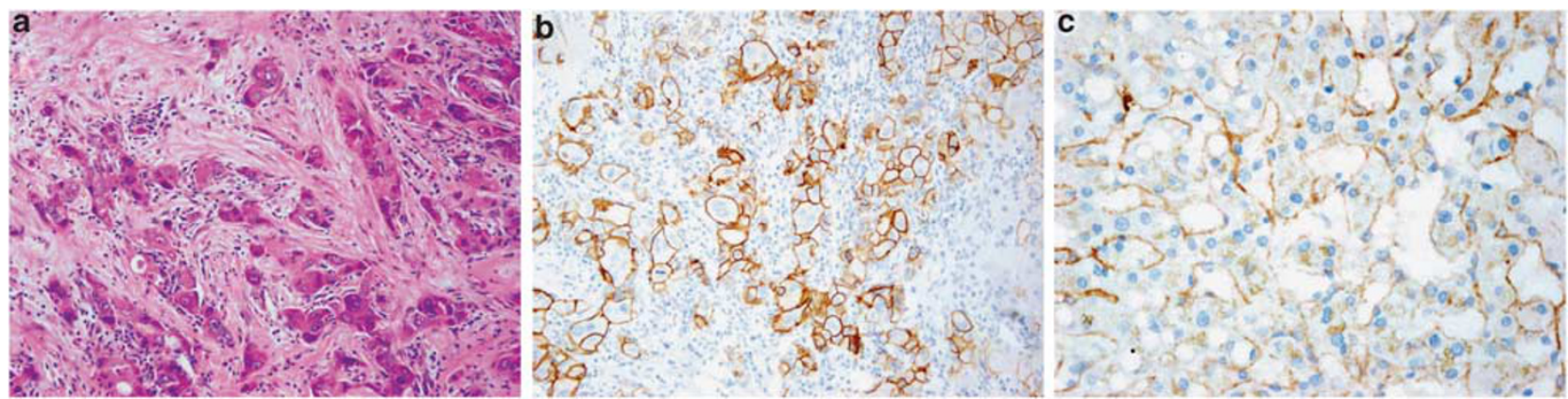

Figure 4 Lack of stem or progenitor cell features in scirrhous hepatocellular carcinoma. (a) Scirrhous hepatocellular carcinoma (hematoxylin-eosin stain, $\times 400$ ). (b) Immunohistochemical stain for CD56 shows focal membranous staining of large neoplastic cells without evidence of small, peripheral progenitor, or stem cell-like cells. (c) Immunohistochemical stain for CD117, showing focal weak membranous staining of large neoplastic cells. Both stains were positive in a small minority of tumor cells.

the cases in our series represent intrahepatic cholangiocarcinoma. However, this premise is not supported by morphological and overall immunohistochemical results. All scirrhous hepatocellular carcinoma cases in our study showed distinct 'hepatoid' morphology and lacked discrete gland formation or mucin production. The expression of GPC3 and/or ARG1 in all the cases also supports the diagnosis of hepatocellular carcinoma.
In some reports, a subpopulation of small tumor cells has been observed at the periphery of the cell nests in scirrhous hepatocellular carcinoma, and it has been postulated that these are likely to represent hepatic stem cells or progenitor cells. In the study by Fujii et $a l,{ }^{45}$ the expression of CK7, CK19, and EPCAM was predominantly observed in these small, peripheral tumor cells, whereas the majority of tumor cells were negative for these markers. 
These cases would correspond to the classical subtype of combined hepatocellular-cholangiocarcinoma with stem cell features in the new WHO classification. ${ }^{5,46}$ In our study, none of the cases showed small tumor cells at the periphery. The staining for CK7, CK19, and EPCAM in our cases was distributed in a patchy or diffuse manner and not restricted to the periphery of tumor cell nests. According to the new WHO classification, the intermediate subtype of combined hepatocellularcholangiocarcinoma with stem cell features also shows abundant fibrous stroma and may closely resemble scirrhous hepatocellular carcinoma. ${ }^{5,47}$ In our cases, the tumor cells did not show morphological features typically associated with progenitor cells, such as small size, oval shape, and scanty cytoplasm. The progenitor-cell-related markers performed on a limited number of cases were also largely negative, further arguing that our cases do not fulfill the criteria for combined hepatocellular-cholangiocarcinoma with stem cell features. ${ }^{5,45}$ CK19 and EPCAM have been considered 'stemness-related' markers in hepatocellular carcinoma. ${ }^{5,44,46-48}$ Although we found high expression of these markers in scirrhous hepatocellular carcinoma, the absence of other 'stemness-related' morphological and immunohistochemical features does not support this interpretation in our series. The significance of this apparent heterogeneity of scirrhous hepatocellular carcinoma across various studies is unclear, but may represent different tumor subgroups and therefore reflect underlying differences in carcinogenetic processes in hepatocellular tumors with abundant fibrous stroma.

Hepatocellular carcinoma with expression of CK7, CK19, and EPCAM has been associated with a poor outcome. ${ }^{48-50}$ As most scirrhous hepatocellular carcinomas express one or more of these markers, it would be expected that scirrhous hepatocellular carcinoma is more aggressive compared with classical hepatocellular carcinoma. However, the outcome of scirrhous hepatocellular carcinoma has been variably reported as better, similar, or worse than classical hepatocellular carcinoma in different studies. ${ }^{2,4,5,7}$ Low numbers of cases and variability in the criteria of diagnosis of scirrhous hepatocellular carcinoma may be responsible for these differences. The low incidence of lymph node metastasis in scirrhous hepatocellular carcinoma compared with intrahepatic cholangiocarcinoma suggests that the biological behavior of scirrhous hepatocellular carcinoma may more closely resemble classical hepatocellular carcinoma. ${ }^{44}$ In addition to these prognostic implications, the inclusion of a cholangiocarcinoma component in the diagnosis can lead to clinical consequences such as use of a gemcitabine-based regimen and denial of transplantation. Until the clinical outcome and therapeutic options in hepatocellular tumors with fibrous stroma are better established, it is prudent to separate scirrhous hepatocellular carcinoma from cholangiocarcinoma and combined hepatocellularcholangiocarcinoma (classical type as well as the type with stem cell features) and rely on the traditional criteria of well-formed glands and mucin production for the diagnosis of a cholangiocarcinoma component. As shown in this and other studies, expression of EPCAM, CK7, and CK19 does not constitute sufficient evidence of biliary differentiation.

In summary, the presence of abundant fibrous stroma, negative HepPar-1 staining, and frequent expression of CK7 and CK19 in scirrhous hepatocellular carcinoma can easily be mistaken for intrahepatic cholangiocarcinoma or metastatic adenocarcinoma. ARG1 and GPC3 are the preferred hepatocellular markers in this morphological setting, and their combined use yielded $100 \%$ sensitivity for scirrhous hepatocellular carcinoma in this series.

\section{Acknowledgements}

The study was funded by the Department of Pathology, University of California, San Francisco.

\section{Disclosure/conflict of interest}

The authors declare no conflict of interest.

\section{References}

1 Okamura N, Yoshida M, Shibuya A, et al. Cellular and stromal characteristics in the scirrhous hepatocellular carcinoma: comparison with hepatocellular carcinomas and intrahepatic cholangiocarcinomas. Pathol Int 2005;55:724-731.

2 Matsuura S, Aishima S, Taguchi K, et al. 'Scirrhous' type hepatocellular carcinomas: a special reference to expression of cytokeratin 7 and hepatocyte paraffin 1 . Histopathology 2005;47:382-390.

3 Kurogi M, Nakashima O, Miyaaki H, et al. Clinicopathological study of scirrhous hepatocellular carcinoma. J Gastroenterol Hepatol 2006;21:1470-1477.

4 Kim SH, Lim HK, Lee WJ, et al. Scirrhous hepatocellular carcinoma: comparison with usual hepatocellular carcinoma based on CT-pathologic features and long-term results after curative resection. Eur J Radiol 2009;69:123-130.

5 Bosman F, Carneiro F, Hruban RH, Theise ND (eds). WHO Classification of Tumours of the Digestive System. IARC Press: Lyon, 2010.

6 Kim SR, Imoto S, Nakajima T, et al. Scirrhous hepatocellular carcinoma displaying atypical findings on imaging studies. World J Gastroenterol 2009;15:2296-2299.

7 Sugiki T, Yamamoto M, Taka K, et al. Specific characteristics of scirrhous hepatocellular carcinoma. Hepatogastroenterology 2009;56:1086-1089.

8 Mosconi S, Beretta GD, Labianca R, et al. Cholangiocarcinoma. Crit Rev Oncol Hematol 2009;69:259-270. 
9 Gatto M, Bragazzi MC, Semeraro R, et al. Cholangiocarcinoma: update and future perspectives. Dig Liver Dis 2010;42:253-260.

10 Choi SB, Kim KS, Choi JY, et al. The prognosis and survival outcome of intrahepatic cholangiocarcinoma following surgical resection: association of lymph node metastasis and lymph node dissection with survival. Ann Surg Oncol 2009;16:3048-3056.

11 Guglielmi A, Ruzzenente A, Campagnaro $\mathrm{T}$, et al. Intrahepatic cholangiocarcinoma: prognostic factors after surgical resection. World J Surg 2009;33: 1247-1254.

12 Llovet JM, Real MI, Montana X, et al. Arterial embolisation or chemoembolisation versus symptomatic treatment in patients with unresectable hepatocellular carcinoma: a randomised controlled trial. Lancet 2002;359:1734-1739.

13 Llovet JM, Ricci S, Mazzaferro V, et al. Sorafenib in advanced hepatocellular carcinoma. N Engl J Med 2008;359:378-390.

14 Valle JW, Wasan H, Johnson P, et al. Gemcitabine alone or in combination with cisplatin in patients with advanced or metastatic cholangiocarcinomas or other biliary tract tumours: a multicentre randomised phase II study-The UK ABC-01 Study. Br J Cancer 2009;101:621-627.

15 Sapisochin G, Fidelman N, Roberts JP, et al. Mixed hepatocellular cholangiocarcinoma and intrahepatic cholangiocarcinoma in patients undergoing transplantation for hepatocellular carcinoma. Liver Transpl 2011;17:934-942.

16 Petrowsky H, Hong JC. Current surgical management of hilar and intrahepatic cholangiocarcinoma: the role of resection and orthotopic liver transplantation. Transplant Proc 2009;41:4023-4035.

17 Mazzaferro V, Regalia E, Doci R, et al. Liver transplantation for the treatment of small hepatocellular carcinomas in patients with cirrhosis. N Engl J Med 1996;334:693-699.

18 Yao FY, Bass NM, Nikolai B, et al. A follow-up analysis of the pattern and predictors of dropout from the waiting list for liver transplantation in patients with hepatocellular carcinoma: implications for the current organ allocation policy. Liver Transpl 2003;9: 684-692.

19 Wee A, Nilsson B. pCEA canalicular immunostaining in fine needle aspiration biopsy diagnosis of hepatocellular carcinoma. Acta Cytol 1997;41:1147-1155.

20 Balaton AJ, Nehama-Sibony M, Gotheil C, et al. Distinction between hepatocellular carcinoma, cholangiocarcinoma, and metastatic carcinoma based on immunohistochemical staining for carcinoembryonic antigen and for cytokeratin 19 on paraffin sections. J Pathol 1988;156:305-310.

21 Borscheri N, Roessner A, Rocken C. Canalicular immunostaining of neprilysin (CD10) as a diagnostic marker for hepatocellular carcinomas. Am J Surg Pathol 2001;25:1297-1303.

22 Shafizadeh N, Ferrell LD, Kakar S. Utility and limitations of glypican-3 expression for the diagnosis of hepatocellular carcinoma at both ends of the differentiation spectrum. Mod Pathol 2008;21: 1011-1018.

23 Yamauchi N, Watanabe A, Hishinuma M, et al. The glypican 3 oncofetal protein is a promising diagnostic marker for hepatocellular carcinoma. Mod Pathol 2005;18:1591-1598.
24 Anatelli F, Chuang ST, Yang XJ, et al. Value of glypican 3 immunostaining in the diagnosis of hepatocellular carcinoma on needle biopsy. Am J Clin Pathol 2008; 130:219-223.

25 Yan BC, Gong C, Song J, et al. Arginase-1: a new immunohistochemical marker of hepatocytes and hepatocellular neoplasms. Am J Surg Pathol 2010; 34:1147-1154.

26 Ramachandran R, Browne WL, Mehdi I, et al. Evidence-based immunohistochemical panel for the distinction of hepatocellular carcinoma and metastatic carcinoma. Mod Pathol 2010;23:370A abstract no. 1645.

27 Ariizumi S, Takasaki K, Otsubo T, et al. Four immunohistochemically different primary liver cancers in one patient. J Gastroenterol 2002;37:750-754.

28 Sugiki T, Yamamoto M, Aruga A, et al. Immunohistological evaluation of single small hepatocellular carcinoma with negative staining of monoclonal antibody hepatocyte paraffin 1. J Surg Oncol 2004;88:104-107.

29 Durnez A, Verslype C, Nevens F, et al. The clinicopathological and prognostic relevance of cytokeratin 7 and 19 expression in hepatocellular carcinoma. A possible progenitor cell origin. Histopathology 2006;49:138-151.

30 Ou-Yang RJ, Hui P, Yang XJ, et al. Expression of glypican 3 in placental site trophoblastic tumor. Diagn Pathol 2010;5:64.

31 Zynger DL, Everton MJ, Dimov ND, et al. Expression of glypican 3 in ovarian and extragonadal germ cell tumors. Am J Clin Pathol 2008;130:224-230.

32 Esheba GE, Pate LL, Longacre TA. Oncofetal protein glypican-3 distinguishes yolk sac tumor from clear cell carcinoma of the ovary. Am J Surg Pathol 2008;32: 600-607.

33 Kandil DH, Cooper K. Glypican-3: a novel diagnostic marker for hepatocellular carcinoma and more. Adv Anat Pathol 2009;16:125-129.

34 Coston WM, Loera S, Lau SK, et al. Distinction of hepatocellular carcinoma from benign hepatic mimickers using glypican-3 and CD34 immunohistochemistry. Am J Surg Pathol 2008;32:433-444.

35 Zhang L, Liu H, Sun L, et al. Glypican-3 as a potential differential diagnosis marker for hepatocellular carcinoma: a tissue microarray-based study. Acta Histochem 2012;114:547-552.

36 Liu H, Li P, Zhai Y, et al. Diagnostic value of glypican-3 in serum and liver for primary hepatocellular carcinoma. World J Gastroenterol 2010;16:4410-4415.

37 Kakar S, Muir T, Murphy LM, et al. Immunoreactivity of Hep Par 1 in hepatic and extrahepatic tumors and its correlation with albumin in situ hybridization in hepatocellular carcinoma. Am J Clin Pathol 2003; 119:361-366

38 Lugli A, Tornillo L, Mirlacher M, et al. Hepatocyte paraffin 1 expression in human normal and neoplastic tissues: tissue microarray analysis on 3,940 tissue samples. Am J Clin Pathol 2004;122:721-727.

39 Baumhoer D, Tornillo L, Stadlmann S, et al. Glypican 3 expression in human nonneoplastic, preneoplastic, and neoplastic tissues: a tissue microarray analysis of 4,387 tissue samples. Am J Clin Pathol 2008;129: 899-906.

40 Yip YC, Wang FH, Vong HT, et al. Significance of glypican-3 immunohistochemistry in diagnosis of hepatocellular carcinoma. Zhonghua Bing Li Xue Za Zhi 2011;40:626-629. 
41 Wang FH, Yip YC, Zhang M, et al. Diagnostic utility of glypican-3 for hepatocellular carcinoma on liver needle biopsy. J Clin Pathol 2010;63:599-603.

42 Shirakawa H, Kuronuma T, Nishimura Y, et al. Glypican-3 is a useful diagnostic marker for a component of hepatocellular carcinoma in human liver cancer. Int J Oncol 2009;34:649-656.

43 Choi S, Park C, Ahn M, et al. Immunohistochemical study of arginase 1 and 2 in various tissues of rats. Acta Histochem 2012;114:487-494.

44 Seok JY, Na DC, Woo HG, et al. A fibrous stromal component in hepatocellular carcinoma reveals a cholangiocarcinoma-like gene expression trait and epithelialmesenchymal transition. Hepatology 2012;55:1776-1786.

45 Fujii T, Zen Y, Harada K, et al. Participation of liver cancer stem/progenitor cells in tumorigenesis of scirrhous hepatocellular carcinoma-human and cell culture study. Hum Pathol 2008;39:1185-1196.
46 Theise ND, Yao JL, Harada K, et al. Hepatic 'stem cell' malignancies in adults: four cases. Histopathology 2003;43:263-271.

47 Kim H, Park C, Han KH, et al. Primary liver carcinoma of intermediate (hepatocyte-cholangiocyte) phenotype. J Hepatol 2004;40:298-304.

48 Kim H, Choi GH, Na DC, et al. Human hepatocellular carcinomas with 'Stemness'-related marker expression: keratin 19 expression and a poor prognosis. Hepatology 2011;54:1707-1717.

49 Uenishi T, Kubo S, Yamamoto T, et al. Cytokeratin 19 expression in hepatocellular carcinoma predicts early postoperative recurrence. Cancer Sci 2003;94: 851-857.

50 Yamashita T, Forgues M, Wang W, et al. EpCAM and alpha-fetoprotein expression defines novel prognostic subtypes of hepatocellular carcinoma. Cancer Res 2008;68:1451-1461. 